\title{
International Space Station Attitude Motion Associated With Flywheel Energy Storage
}

\author{
Carlos M. Roithmayr \\ NASA Langley Research Center, Hampton, Virginia, 23681 \\ 757-864-6778; c.m.roithmayr@larc.nasa.gov
}

\begin{abstract}
Flywheels can exert torque that alters the Station's attitude motion, either intentionally or unintentionally. A design is presented for a once planned experiment to contribute torque for Station attitude control, while storing or discharging energy. Two contingencies are studied: the abrupt stop of one rotor while another rotor continues to spin at high speed, and energy storage performed with one rotor instead of a counter rotating pair. Finally, the possible advantages to attitude control offered by a system of ninety-six flywheels are discussed.
\end{abstract}

\section{INTRODUCTION}

The International Space Station (ISS) Payloads Office, through Johnson Space Center's Engineering and Research Technology Program, has for the past two years funded a program at Glenn Research Center to develop flywheel energy storage technology. What began as an experiment planned to demonstrate integrated energy storage and attitude control has evolved into a project that may add significantly to the capacity for energy storage onboard the Station, and reduce or eliminate the cost and time required to replace chemical batteries. The resulting technology could be applied by the industrial participants of this program in future spacecraft, or in a wide variety of other areas.

Energy storage and attitude control are accomplished with two separate devices on present spacecraft. Batteries are typically used to store and supply electrical energy produced by photovoltaic cells; however, batteries are quite massive, and battery life often limits the life of a spacecraft. Spinning cylinders, such as reaction wheels and Control Moment Gyroscopes (CMGs), are often employed to control orientation without expending propellant. Although these devices possess significant rotational kinetic energy, there are no provisions to convert it back into electrical form. Mechanical flywheel systems are an attractive alternative to batteries. Their longevity is superior, and they are less massive than batteries; moreover, flywheels can simultaneously store energy and control attitude, making it possible to reduce spacecraft mass even further.

Each device in the ISS Flywheel Energy Storage System (FESS) [formerly the Attitude Control and Energy Storage Experiment (ACESE)] will consist of two counter-rotating rotors placed in vacuum housings, and levitated with magnetic bearings. Motor-generators will connect the rotors to the existing electrical power system so that they can store energy when it is available from the photovoltaic arrays, and supply energy when it is needed. Each rotor, made up of a metallic hub and a rim of composite material, will be approximately $28 \mathrm{~cm}$ in diameter and $38 \mathrm{~cm}$ in length, and spin at angular speeds ranging between 18,000 and 60,000 rev/min. Whereas the ACESE was to have been performed with one pair of rotors, as many as 48 pairs could make up the FESS on the Assembly Complete configuration of ISS.

Through counter-rotation it is possible to store energy in (or retrieve energy from) a pair of rotors without exerting torque on the spacecraft. Of course, flywheels are put to greatest advantage when the application of torque for attitude control, and energy storage are conducted concurrently. The departure of a rotor pair from the condition of counter rotation can thus be intentional, or it can be inadvertent when there is a failure of some part of the flywheel system. In either case, torque is exerted and it is important to analyze the resulting attitude motion of the Station, together with the behavior of the Station's CMGs. The results of several simulations addressing this need in connection with the ACESE 
are reported in this paper, followed by a preliminary study of what could be accomplished by the FESS in providing attitude control.

Before proceeding to the analysis results, brief descriptions are given of the simulation program used, the model of a flywheel pair, and the algorithm for calculating flywheel motor torques.

\section{SIMULATION DESCRIPTION}

The numerical results reported herein were obtained with the Space Station Multi Rigid Body Simulation [SSMRBS; see (Anonymous, 1999)], a computer program that numerically integrates equations of motion governing the behavior of a spacecraft modeled as a rigid, multibody, multi-degree-of-freedom system.

SSMRBS is used primarily to evaluate the performance of candidate attitude control schemes for Space Station, and to validate Guidance, Navigation, and Control systems for each stage of the Station assembly sequence. Analysts are able to study the interplay of attitude control with other Space Station activities such as collection of solar power, radiation of excess heat, remote manipulator operations, centrifuge operations, and microgravity experiments.

Simulations typically involve, simultaneously, the control of core body attitude with a CMG momentum manager or a reaction control system (or both); independent feedback control of each solar array and radiator joint angle; and transportation of payloads with a mobile transporter or remote manipulator.

\section{Model of a Flywheel Pair}

All bodies are treated as rigid in the simulations to be discussed; a flywheel rotor is considered to be a right circular cylinder whose mass is distributed uniformly. The model of the physical connection between a rotor and the outboard truss that is simplest and most appropriate for the study of the Station's attitude behavior is a revolute, or hinge joint whose axis is coincident with the rotor's longitudinal central principal axis of inertia, or spin axis. In such a model of the joint, the spin axis of the rotor is fixed in the outboard truss and in the rotor; that is, the model does not account for any changes in the spin axis direction relative to the outboard truss that are in fact allowed by the magnetic bearings, nor does it permit any coning motions of the rotor. Counter rotation of the two rotors requires that their spin axes be parallel.

Relative motion between a body and its inboard body can be brought about in the simulations by means of a motor; the masses of the two bodies are considered to include the mass of the motor parts. The inboard body exerts torque on the outboard body through the motor, and in accordance with the law of action and reaction, torque of equal magnitude and opposite direction is applied to the inboard body. A flywheel unit's motor-generator is represented by such a motor, whose detailed electrical behavior need not be modeled.

Each flywheel pair $F$ is made up of rotors $A$ and $B$, attached to an outboard truss $C$ such that their spin axes are parallel to a unit vector $\hat{\boldsymbol{\lambda}}$ fixed in $A, B$, and $C$. Changes in the angular speeds of $A$ and $B$ relative to $C$ are determined by the following three scalars:

$$
T^{C / A} \triangleq \mathbf{T}^{C / A} \cdot \hat{\boldsymbol{\lambda}}, \quad T^{C / B} \triangleq \mathbf{T}^{C / B} \cdot \hat{\boldsymbol{\lambda}}, \quad T^{C / F} \triangleq \mathbf{T}^{C / F} \cdot \hat{\boldsymbol{\lambda}}
$$

where the torque exerted by $C$ on $A$, and $B$, are denoted by the vectors $\mathbf{T}^{C / A}$, and $\mathbf{T}^{C / B}$, respectively. The torque $\mathbf{T}^{C / F}$ exerted by $C$ on $F$ is simply the sum of $\mathbf{T}^{C / A}$ and $\mathbf{T}^{C / B}$. Expressions for the first two scalars, or "motor torques", are derived in (Roithmayr, 1999), and are written as:

$$
\begin{gathered}
T^{C / B}=\frac{{ }^{C} P^{F}-T^{C / F} u_{A}}{u_{B}-u_{A}}, \\
T^{C / A}=T^{C / F}-T^{C / B},
\end{gathered}
$$

where ${ }^{C} P^{F}$ is the power of $F$ in $C$, or rate of change of rotational kinetic energy, an important measure of energy storage. When ${ }^{C} P^{F}>0$, the flywheels are charging; that is, rotational kinetic energy of $F$ in $C$ is increasing. Time histories of ${ }^{C} P^{F}$ and $T^{C / F}$ are specified by the analyst. $u_{A}$, and $u_{B}$, are angular speeds in $C$ of $A$, and $B$, respectively. 


\section{ATTITUDE CONTROL AND ENERGY STORAGE EXPERIMENT}

The plan for the ACESE was to attach a single pair of flywheels to the Station's starboard outboard truss at an unoccupied site for a battery charge-discharge unit and two batteries, and operate it in combination with existing batteries. The primary objective of the experiment was to demonstrate energy storage, whereas the secondary objective was to use flywheels to exert torque on the Station, and show measurable evidence that torque had been applied. In the interest of simplicity, the time history of $T^{C / F}$ was to be predetermined rather than governed by a feedback control scheme, and the existing Station attitude control system was not to be modified to account for presence of the flywheels. The design of ACESE hardware was simplified considerably by omitting devices for measuring directly the torque applied by the experiment to the Station. Measurements were, instead, to be obtained indirectly by examining telemetry of CMG torque and momentum, or flywheel rotor angular speeds.

In the remainder of this section, the time histories of $T^{C / F}$ and ${ }^{C} P^{F}$ that were proposed to meet the secondary objective of the experiment are spelled out, followed by a summary of simulation predictions regarding the behavior of the Station's attitude and the CMGs. Next, simulation results are reported for two hypothetical malfunctions that would affect the storage or discharge of energy.

The amount of angular momentum that can be stored in one pair of flywheels makes it difficult to demonstrate attitude control in a convincing fashion; by the same token, the two malfunctions considered here have very little effect on the attitude motion of the Station. The magnitude of central angular momentum in an inertial reference frame that each CMG can possess is limited to 4,745 N-m-s; when all four CMGs are operating, the magnitude of the resultant of the angular momenta can reach 18,981 N-m-s. In comparison, one flywheel rotor possesses approximately 1,573 N-m-s of angular momentum at high speed $\left(0.3010 \mathrm{~kg}-\mathrm{m}^{2} \times 50,000 \mathrm{rev} / \mathrm{min}\right)$; that is, about $\frac{1}{3}$ of the momentum of a CMG.

\section{Flywheel Torque Assist for CMGs}

The goal of demonstrating attitude control with the ACESE was to have been met by employing a sinusoidal time history of $T^{C / F}$ in order to assist the CMGs in their job of controlling the orientation of the Station core body, maintaining a torque equilibrium attitude.

The Space Station is modeled as a collection of rigid bodies fastened together: a core body, outboard truss structures, solar arrays, radiators, and so forth. Analysis of the ACESE is performed with configuration c080_13a, comprising 15 bodies whose mass distributions and topography are described in (Anonymous, 1998). Two additional bodies attached to the starboard outboard truss represent the flywheel rotors. The masses of $A$ and $B$ are given by $m_{A}=m_{B}=31.67$ $\mathrm{kg}$, and the central inertia dyadics are given by $\underline{\mathbf{I}}^{A / A^{\star}}=\underline{\mathbf{I}}^{B / B^{\star}}=0.3986 \hat{\mathbf{a}}_{1} \hat{\mathbf{a}}_{1}+0.3010 \hat{\boldsymbol{\lambda}} \hat{\boldsymbol{\lambda}}+0.3986 \hat{\mathbf{a}}_{3} \hat{\mathbf{a}}_{3} \mathrm{~kg}-\mathrm{m}^{2}$, where $\hat{\mathbf{a}}_{1}$ and $\hat{\mathbf{a}}_{3}$ are any two unit vectors perpendicular to each other and to $\hat{\boldsymbol{\lambda}}$. Unit vector $\hat{\boldsymbol{\lambda}}$ has the same direction as $\hat{\mathbf{c}}_{2}$, a unit vector fixed in the starboard outboard truss (and in the core body) in the Station's "y" or starboard direction, parallel to the axis of the revolute joint (known as the alpha joint) that connects the outboard truss to the Station core body.

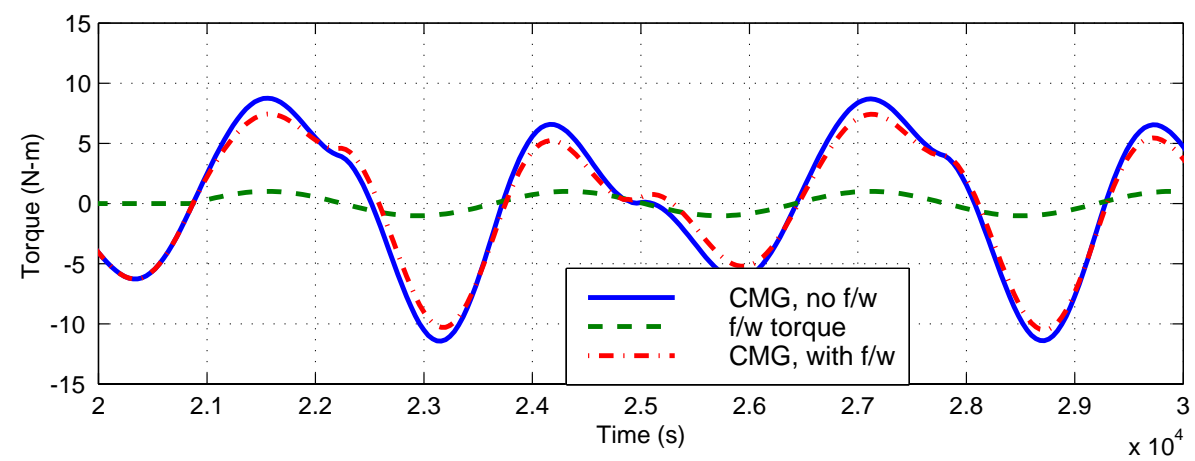

FIGURE 1. CMG Torque, Flywheel Torque $\left(T^{F / C}\right)$. 
Simulation results for Station configuration 13A, in the absence of flywheel torque $\left(T^{C / A}=T^{C / B}=0\right)$, are shown in Fig. 1 for a time interval during which attitude motion has reached steady-state; the torque exerted by the CMGs on the core body, projected into the $\hat{\mathbf{c}}_{2}$ direction, is displayed with the solid curve. Inspection reveals this curve to be nearly a simple sinusoid; the orbital period of the Station is in this case approximately 5,560 s, so the sinusoid has a frequency of two cycles per orbit. Consequently, the form chosen for $T^{C / F}$ is:

$$
T^{C / F}=\tau \sin 2 \omega\left(t-t^{\star}\right),
$$

where $\omega$ is the frequency at which the Station orbits the Earth, and $t^{\star}$ is a particular value of the time $t$ at which the solid curve reaches zero, and has a positive slope. Limits on the angular speeds $u_{A}$ and $u_{B}$ of the rotors, together with the value of the axial central principal moment of inertia of the rotors, effectively limits the absolute value of $\tau$ to 1.017 $\mathrm{N}$-m. A plot of $T^{F / C} \triangleq-T^{C / F}$ is displayed in Fig. 1 with a dashed curve, where $T^{C / F}$ is given by Eq. (4) with $\tau=-1.017 \mathrm{~N}-\mathrm{m}, \omega=0.0011295 \mathrm{rad} / \mathrm{s}$, and $t^{\star}=20,860 \mathrm{~s}$. Prior to $t=t^{\star}, \tau=0$.

A simulation is performed in which the flywheels assist the CMGs and store energy at the same time; $T^{C / B}$ and $T^{C / A}$ are calculated according to Eqs. (2) and (3) for $t \geq t^{\star} .{ }^{C} P^{F}$ is specified as 2,200 watts during the sunlit portion of an orbit, and as $-1.57 \times 2,200$ watts during eclipse. (In these particular simulations, the ratio of time spent by the spacecraft in sunlight to that spent in eclipse is 1.57.) $T^{F / C}$ is specified as indicated in Fig. 1 with the dashed curve, and the resulting reduction in CMG torque is shown with a dash-dot curve. The reduction in CMG torque is reflected in a smaller magnitude of the resultant of central angular momenta in inertial space of the four CMGs (not shown); the decrease in momentum magnitude periodically reaches $270 \mathrm{~N}-\mathrm{m}-\mathrm{s}$.

The CMG momentum management scheme used in these simulations places greater emphasis on controlling orientation than on minimizing CMG momentum; therefore, one expects to see only small differences in the roll, pitch, and yaw of the core body with respect to, say, a local-vertical-local-horizontal reference frame. Such expectations are in fact borne out; of the three orientation angles, the largest difference occurs in pitch and is less than $0.18 \mathrm{deg}$, and is transient in nature.

\section{Energy Storage Contingencies}

Ideally, the storage and discharge of energy takes place as the rotors are counter rotating $\left(u_{A}=-u_{B}\right)$, and no torque is applied by the flywheel pair to the outboard truss $C$. However, malfunctions can occur in which the rotors no longer counter rotate, and $T^{F / C}$ thus differs from 0 . One such situation involves having to bring a rotor to an abrupt stop while the other rotor continues to spin. A second condition of this kind occurs when energy storage must be performed with one rotor because it is not possible to operate the second rotor. Both of these situations are examined in this subsection.

\section{Emergency Shutdown of One Rotor}

An abrupt stop of one rotor is simulated by starting with initial values $u_{B}(t=0)=-u_{A}(t=0)=50,000 \mathrm{rev} / \mathrm{min}$, setting $T^{C / A}=T^{C / B}=0$ for $0 \leq t<20,860 \mathrm{~s}$, and then making $T^{C / B}=-2.7 \mathrm{~N}-\mathrm{m}$ for $20,860 \leq t \leq 21,441$ s. In this way $B$ is brought to rest in $C$, while the angular speed $u_{A}$ remains equal to $-50,000 \mathrm{rev} / \mathrm{min}$. As in the previous section, results are compared to those of the simulation in which $T^{C / A}=T^{C / B}=0$ at all times, and $u_{A}(t=0)=u_{B}(t=0)=0$. Several parameters are examined, and the greatest differences observed in each are reported in Table 1. "Attitude, wrt LVLH" refers to the orientation of the Station core body relative to a local-verticallocal-horizontal reference frame, described with a body-three, 3-2-1 (yaw, pitch, roll) rotation sequence. Differences are shown for the resultant of the four $\mathrm{CMG}$ central angular momenta, and the resultant CMG torque, projected on to $\hat{\mathbf{b}}_{1}, \hat{\mathbf{b}}_{2}$, and $\hat{\mathbf{b}}_{3}$, a set of right-handed, mutually perpendicular unit vectors fixed in the Station's core body. In addition, the difference in magnitude of the CMG momentum is shown in the final column. The differences in attitude and CMG torque are not significant. 
TABLE 1. Differences Observed as a Result of Rotor Shutdown.

\begin{tabular}{|l|c|c|c|c|}
\hline Parameter & Yaw & Pitch & Roll & \\
\cline { 1 - 4 } Attitude, wrt LVLH (deg) & 0.023 & 0.350 & 0.035 & \\
\hline & $\cdot \hat{\mathbf{b}}_{1}$ & $\cdot \hat{\mathbf{b}}_{2}$ & $\cdot \hat{\mathbf{b}}_{3}$ & Magnitude \\
\hline CMG Angular Momentum (N-m-s) & 100 & 2,170 & 135 & 1,150 \\
\hline CMG Torque (N-m) & 0.16 & 4.5 & 0.26 & - \\
\hline
\end{tabular}

\section{Energy Storage Using a Single Rotor}

Should one rotor become incapacitated, it may be possible to demonstrate energy storage at a reduced level with the remaining rotor. With $A$ regarded as the malfunctioning rotor, the event is simulated by setting $T^{C / F}$ to 0 in Eq. (2), and by specifying ${ }^{C} P^{F}$ as 1,100 watts when the spacecraft receives sunlight, and as $-1.57 \times 1,100$ watts when the spacecraft is in darkness. Instead of calculating $T^{C / A}$ according to Eq. (3), it is defined to be 0 throughout the simulation.

The particular initial values of orbit parameters and position of the sun in these simulations make it convenient to choose $u_{B}(t=0)=38,000 \mathrm{rev} / \mathrm{min}$; thereafter, $u_{B}$ varies between 51,000 and $19,000 \mathrm{rev} / \mathrm{min}$. Since $A$ is to be at rest in $C, u_{A}$ is chosen as 0 at $t=0$, and remains exceedingly small for the duration of the simulation.

As before, results are compared to those of the simulation in which $T^{C / A}=T^{C / B}=0$ at all times, and the greatest differences observed are reported in Table 2. The differences seen in attitude and CMG torque are not significant.

TABLE 2. Differences Observed during Single-Rotor Energy Storage.

\begin{tabular}{|l|c|c|c|c|}
\hline Parameter & Yaw & Pitch & Roll & \\
\hline Attitude, wrt LVLH (deg) & 0.010 & 0.140 & 0.019 & \\
\hline & $\cdot \hat{\mathbf{b}}_{1}$ & $\cdot \hat{\mathbf{b}}_{2}$ & $\cdot \hat{\mathbf{b}}_{3}$ & Magnitude \\
\hline CMG Angular Momentum (N-m-s) & 75 & 880 & 135 & 407 \\
\hline CMG Torque (N-m) & 0.16 & 1.6 & 0.16 & - \\
\hline
\end{tabular}

\section{FLYWHEEL ENERGY STORAGE SYSTEM ON ASSEMBLY COMPLETE}

Consideration is being given to replacing many or all of the chemical batteries with flywheels on board the Assembly Complete configuration. A complete replacement would entail $N=96$ rotors arranged in 48 counter-rotating pairs. If each rotor has an axial central principal moment of inertia of $J=0.3010 \mathrm{~kg}-\mathrm{m}^{2}$, and an allowable angular speed range between $\omega_{l}=20,000 \mathrm{rev} / \mathrm{min}$ and $\omega_{u}=60,000 \mathrm{rev} / \mathrm{min}$, then the total useable angular momentum is $N\left[J\left(\omega_{u}-\omega_{l}\right)\right]$ $=121,000 \mathrm{~N}$-m-s. If one assumes that only $25 \%$ to $50 \%$ of this figure is available for attitude control (the remainder being reserved for energy storage), this leaves 30,250 to $60,500 \mathrm{~N}-\mathrm{m}-\mathrm{s}$, or 1.5 to 3 times the amount available from the current set of 4 CMGs.

Each flywheel pair will be mounted in either the starboard or the port outboard truss structure. It is geometrically possible to orient some of the pairs so that their spin axes have the same direction as $\hat{\mathbf{c}}_{3}$, a unit vector perpendicular to $\hat{\mathbf{c}}_{2}$ and fixed in the outboard truss, marking the " $\mathrm{z}$ " direction. (It is not possible to mount the spin axes in the $\hat{\mathbf{c}}_{1}$, or outboard truss " $x$ " direction.) Analysis of a 48-pair FESS is performed with the 17-body Station configuration c113_ac (Assembly Complete) described in (Anonymous, 1998). A reasonable distribution of orientations would make $\hat{\mathbf{c}}_{2}$ the direction of the spin axes for 24 pairs, and $\hat{\mathbf{c}}_{3}$ the direction of the other 24 . In the interest of simplicity it is assumed that every pair in the former set of 24 behaves identically, and likewise for the latter set of 24; therefore, the former set is modeled as a single pair whose mass properties are 24 times the values given above for the ACESE, and likewise for the latter set.

A simple demonstration of the use of FESS for energy storage and attitude control has been performed. A simulation of motion of Assembly Complete (without flywheels) yields the torque exerted by CMGs as they maintain steady-state 
torque equilibrium attitude. The $\hat{\mathbf{c}}_{2}$ and $\hat{\mathbf{c}}_{3}$ measure numbers of CMG torque are then curve fit with Fourier series in order to command flywheel torque $\left(T^{F / C}\right)$ for the corresponding 24-pair sets in a second simulation. ${ }^{C} P^{F}$ for each set is specified as $-105.6 \mathrm{kw}(24 \times-4,400$ watts) for discharge during eclipse, and $105.6 \mathrm{kw} / 1.57=67.3 \mathrm{kw}$ during sunlight. Thus, the entire FESS discharges at $211.2 \mathrm{kw}$, and charges at $134.6 \mathrm{kw}$.

The magnitude of CMG momentum is shown for the two simulations in Fig. 2; the dashed curve indicates results in the absence of flywheel torque, and the solid curve shows the momentum magnitude when the flywheels are used to assist the CMGs and store energy. A comparison of the two curves indicates that CMG momentum magnitude can be reduced by as much as $7,000 \mathrm{~N}-\mathrm{m}-\mathrm{s}$.

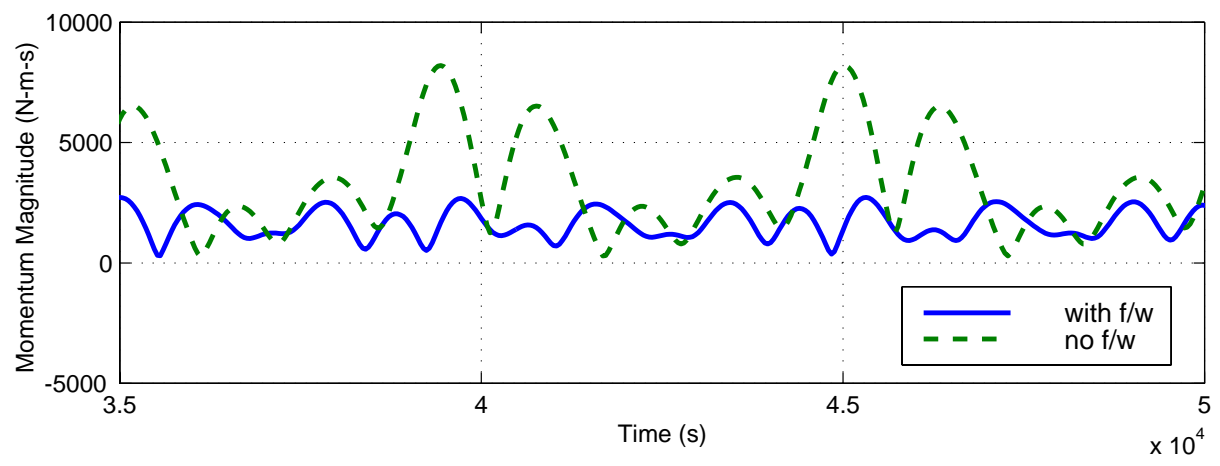

FIGURE 2. CMG Momentum magnitude.

\section{CONCLUSIONS}

The use of a single system of flywheels to perform energy storage and attitude control is a promising alternative to the current practice of employing two separate systems. The secondary objective of the ACESE, to contribute torque for Station attitude control while storing or discharging energy, could have been achieved by matching the phase of the sinusoidal torque exerted by CMGs when attitude motion reaches steady state. Two possible contingencies (the abrupt stop of one rotor while the other rotor continues to spin at high speed, and energy storage carried out with one rotor instead of a counter rotating pair) are seen to have negligible effects on the behavior of attitude and the CMGs. Finally, a flywheel energy storage system placed on the ISS at Assembly Complete and composed of 48 pairs of flywheels can provide angular momentum for attitude control that is 1.5 to 3 times the amount available from the current CMG system.

\section{ACKNOWLEDGMENTS}

This research was sponsored, in part, by the Advanced Programs Office, Code M, at NASA Headquarters.

\section{REFERENCES}

Anonymous, International Space Station On-Orbit Assembly, Modeling, and Mass Properties Databook; Design Analysis Cycle \#7, Revision D Assembly Sequence, JSC-26557, Rev. I, Vol. 1, NASA Johnson Space Center, Houston, TX, September, 1998.

Anonymous, Space Station Multi Rigid Body Simulation User's Guide, version b2.2, EG-UG-28702, NASA Johnson Space Center, Aeroscience and Flight Mechanics Division, Houston, TX, June, 1999.

Roithmayr, C. M., International Space Station Attitude Control and Energy Storage Experiment: Effects of Flywheel Torque, NASA TM-1999-209100, NASA Langley Research Center, Hampton, VA, February, 1999. 